\title{
Review of High Energy Density Physics Activity in Europe
}

\author{
Julien FUCHS, ${ }^{1}$ Bruno ALBERTAZZI, ${ }^{1}$ Jesús Álvarez RUIZ, ${ }^{2}$ Stefano ATZENI,${ }^{3}$ A. BENUZZI-MOUNAIX, ${ }^{1}$ \\ Claude BONIFACE, ${ }^{4}$ Erik BRAMBRINK, ${ }^{1}$ A. CIARDI, ${ }^{5}$ Emmanuel D'HUMIÈRES,${ }^{6}$ L. A. GIZZI, ${ }^{7}$ \\ M. HARMAND,${ }^{8}$ S. HULIN,${ }^{6}$ Paul MCKENNA, ${ }^{9}$ J. L. MIQUEL,${ }^{4}$ M. KOENIG, ${ }^{1}$ A. RAVASIO, ${ }^{1}$ \\ Steven James ROSE, ${ }^{10}$ Markus ROTH, ${ }^{11}$ Vladimir TIKHONCHUK, ${ }^{6}$ and Nigel WOOLSEY ${ }^{12}$ \\ ${ }^{1}$ LULI, Ecole Polytechnique, CNRS, CEA, UPMC, route de Saclay, 91128 Palaiseau, France \\ ${ }^{2}$ Instituto de Fusión Nuclear, Univesidad Politécnica de Madrid, Madrid, 28006, Spain \\ ${ }^{3}$ Dip. SBAI, Università di Roma La Sapienza and CNISM, Italy \\ ${ }^{4}$ CEA/DAM Ile de France, Bruyères le Châtel, 91297 Arpajon Cedex, France \\ ${ }^{5}$ LERMA, Observatoire de Paris, Ecole Normale Supérieure, \\ Université Pierre et Marie Curie, UMR 8112 CNRS, 5 Place Jules Jannsen, 92195, Meudon, France \\ ${ }^{6}$ Univ. Bordeaux - CEA - CNRS, CELIA, UMR 5107, F-33405 Talence, France \\ ${ }^{7}$ ILIL at National Institute of Optics, CNR, and INFN Pisa, Italy \\ ${ }^{8}$ DESY, Notkestr. 85, 22607 Hamburg, Germany \\ ${ }^{9}$ SUPA, Department of Physics, University of Strathclyde, Glasgow G4 ONG, UK \\ ${ }^{10}$ Plasma Physics Group, The Blackett Laboratory, Imperial College London, Prince Consort Road, London, SW7 2AZ, UK \\ ${ }^{11}$ T. U. Darmstadt, Germany \\ ${ }^{12}$ York Plasma Institute, University of York, York, UK
}

(Received October 29, 2012)

\begin{abstract}
Significant efforts are being conducted in various areas of high-energy-density (HED) science in Europe. In this frame, we will below review some recent results obtained in specific topics. In particular, we will outline recent experimental and theoretical results obtained in various fields as atomic physics, warm dense matter (matter with density above or equal solid, and temperatures above or equal to $1 \mathrm{eV}$ ), laserplasma interactions, the production of compact particle sources, magnetized plasmas, with relevance to varied applications like inertial confinement fusion (ICF), geophysics or astrophysics.
\end{abstract}

Key Words: High-energy-density matter, Inertial confinement fusion, Geophysics, Astrophysics, Particle acceleration

\section{Introduction}

The possibility of producing, in a controlled way, matter at solid density $\left(1-10 \mathrm{~g} / \mathrm{cm}^{3}\right)$, compressed or not, while maintaining it at high temperature $(1-100 \mathrm{eV})$ has been a goal for a long time ${ }^{1)}$ as this class of states of matter of extremely high energy density (HED) is relevant to a wide range of disciplines. The peculiar interest of such extreme states of matter is not only that they were previously unattainable in laboratory experiments, but also that that they are rich in new physics phenomena and compelling applications. ${ }^{2)}$ At the boundary between condensed matter and plasma physics, such HED matter currently involves many laboratories worldwide. The wide interest it attracts comes from the fact that is found not only in astrophysical objects but also in the transition from solid density matter to the plasma state found in numerous plasma generators and in the initial low-adiabat compression phase of indirectly driven inertial fusion experiments. Accurate modeling of inertial plasmas requires physical data, equation of states and transport coefficients in this HED regime where classical theories such as plasma kinetic theory and condensed matter theory fail, as it is defined by ion-ion correlation and Fermi degeneracy parameters that are both on the order of the unity or greater. Below, we will review some aspects of HED science, highlighting examples of recent experimental and theoretical results obtained across Europe.

\section{Material Science}

Despite having been the subject of much effort, progress in accurate atomic physics description of ICF materials is still necessary in order to improve our understanding and predictive capabilities, as witnessed by the present difficulties encountered by the ignition campaign at NIF (USA). In the UK, non local-thermal-equilibrium (LTE) atomic kinetics codes have been used in order to investigate carbon and silicon in regimes relevant to the ablator on NIF capsules. We have discussed the effects of the significant departures from LTE at below-solid density and of the full treatment of the incident radiation field including the interaction between the radiation 
and the atomic kinetics when considering a highly detailed absorption and emission spectrum such as the one shown in Fig. 1. ${ }^{3)}$

Still regarding ICF capsule materials, progress on the generation and characterization of carbon in the warm dense matter (WDM) regime has been made in Germany. Graphite and diamond samples were irradiated with intense laser accelerated proton beams in experiments conducted at the Trident laser at LANL and the Vulcan laser at RAL reaching temperatures around $10000 \mathrm{~K}$. Via the scattering of laser-generated X-ray line radiation, the microscopic structure of these exotic matter states could be determined. In a different approach, warm dense carbon was produced by shock-compression of graphite in experiments at the GSI Helmholtzzentrum für Schwerionenforschung. Using spectrally resolved X-ray scattering, the solid liquid transition of carbon in a pressure regime around $100 \mathrm{GPa}$ was directly measured. The recent availability of Free Electron Lasers (FEL) X-ray sources offers also new perspectives for WDM research. For example, X-ray scattering pump-probe experiment can now be performed with high temporal resolution to study the electron-ion equilibration processes where the typical time scales are on the order of several 100's of femtoseconds to several picoseconds. Such experiments exploit X-ray scattering as an alternative diagnostic method for probing dense systems. ${ }^{4}$ This technique, which is the X-ray analogue of optical Thomson scattering, allows us to determine directly thermodynamic properties and dynamic structure factors of dense systems where optical light cannot penetrate. Using it, in recent experiments at FLASH, it was possible to follow the dynamics of liquid hydrogen heated by optical laser or FEL pulses by probing the heated sample with $13.5 \mathrm{~nm}$ XUV FEL radiation at different time delays.

Significant research is also taking place on materials for inertial confinement fusion on the experimental and theoretical fronts in Spain. A design of plasma facing components, in particular those for the first wall, has been undertaken by studying the interaction of high fluxes of energetic ion bursts produced in the fusion reactions with fusion suited materials (refractory metals such as Tungsten; carbon based materials such as carbon composites and nanodiamond, etc). For this, ultra intense laser systems are used to reproduce intense ion beams, and experiments are performed to irradiate samples with proton, deuteron and carbon beams. ${ }^{6}$ ) The study of systems at very high pressure is also carried out by simulations using first principles and Molecular Dynamics. ${ }^{7)}$ Ab initio modelling codes such as SIESTA, Ab Init and Quatum Expresso are employed to investigate the behavior of hydrogen isotopes at pressures above $1 \mathrm{GPa}^{8,9), \uparrow}$

Significant progress have been also made on studying iron under high pressure, a topic of particular interest for geophysics, as the core of earth like planets is mainly constituted of iron with small fractions of impurities. Especially the melting curve is determining the core dynamics, as the crystallization of liquid iron in the core is the major energy source in the planetary core. With the discovery of extrasolar planets having a similar composition to planets in the solar system but much larger sizes, the research into iron properties at even higher pressures has entered the focus of attention. Thus, exploring the iron phase diagram up to pressures of $15 \mathrm{Mbar}$ and $10000 \mathrm{~K}$ is a key for planetary science. As neither shocks nor static compression can reach these parameters, the isentropic compression scheme has become an important technique in the recent years. Isentropic compression with lasers can be achieved by increasing smoothly the laser intensity over several ns and thus compressing quickly without generating a shock. Systematic investigation of the phase transition dynamics of the iron alpha-epsilon transition and second the shock-less compression of iron up to multi-Mbar pressures has been performed at LULI.

Figure 2 shows interface velocity profiles recorded with a VISAR. In an isentropic scheme, these velocities can be attributed to pressure, thus these graphs show the pressure history of the sample. The plateau in the curve is characteristic for the phase transition, more precisely for the time, where both phases coexist. As the specific volume is reduced during the transition, the material softens and the pressure stays constant. The length of the plateau (not fully shown here) is related to the reaction rate, thus the time the transformation to the new phase is completed.

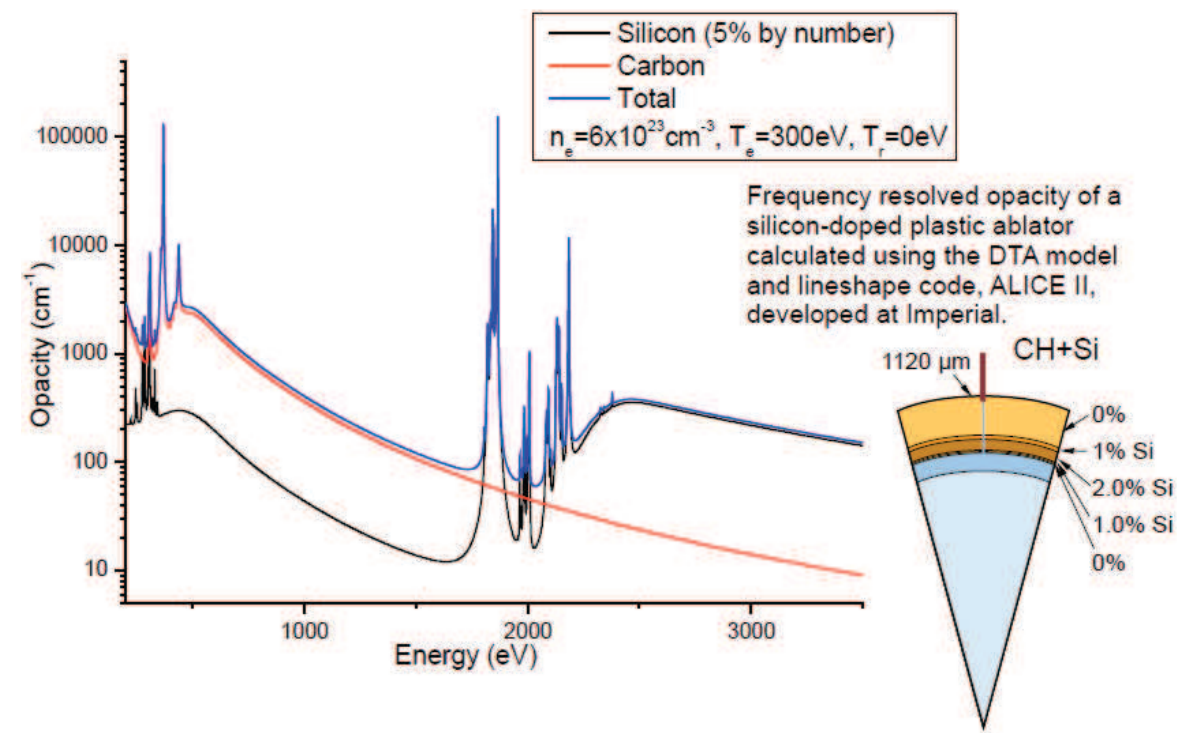

Fig. 1 The frequency resolved opacity for a Carbon/Silicon mix calculated with ALICE II. The Silicon is included at 5\% by number.

${ }^{\dagger}$ URL: http://webdav.luli.polytechnique.fr/public/HiPER-Bordeaux2012/ 




Fig. 2 Interface velocity profiles obtained during ramp compression of iron, showing the alpha-epsilon transition.

Finally, progress has also been made on the topic of ion stopping in plasmas, a point of importance of ICF. Directly driven laser targets and hohlraum driven targets were used in Germany to provide plasma targets for energy loss measurements of swift heavy ions in plasma. A new model was developed, which does no longer relies on mean charge states was found to be in excellent agreement with the experimental data. In a further development, ions at energies relevant for alpha particle heating in fusion targets have been tested. In France, measurements of single shot mean charge equilibration of carbon beams generated by high intensity short pulse lasers were performed in cold or isochorically heated solid density aluminum matter. ${ }^{10)}$ Plasma effects were shown, in such matter heated up to $1 \mathrm{eV}$, not to significantly impact the equilibration of carbon ions with energies $0.045-0.5 \mathrm{MeV} /$ nucleon, and to allow for a first evaluation of semi-empirical formulas or abinitio models that are being used to predict the equilibrium mean charge state for light ions passing through warm dense matter.

\section{Investigation of ICF designs}

\subsection{Shock ignition}

As an alternative to the standard indirect-drive approach to ICF which is pursued at NIF (USA) and LMJ (France), shock ignition ${ }^{11)}$ is being studied as an option for achieving high target gains. The scheme is based on the separation of the two phases: compression by the long pulses and ignition triggered by a strong convergent shock launched at the end of the shell implosion with a high intensity spike. This concept is very promising because radiation hydrodynamic simulations predict high gain and it does not require any complex cone-in-ashell target or high-power unconventional lasers like for Fast Ignition.

A group at the University of Roma has studied several aspects of the design of shock ignition targets using analytical models, and one- and two-dimensional hydrodynamics fluid codes. In the frame of the HiPER project, the group has first ${ }^{12)}$ analyzed the robustness of a target designed earlier for fast ignition and then showed ${ }^{13)}$ to be suitable for shock ignition, too. Aspects considered were i) 1D sensitivity to parameter changes, ii) control of Richtmyer-Meshkov and RayleighTaylor hydrodynamic instabilities, iii) effects of non uniform irradiation and of target misplacement. Subsequently, the group has studied how the considered target scales at larger size (and energy) ${ }^{14)}$ and with the laser wavelength, ${ }^{15)}$ and has generated gain curves. Current studies concern model improvements (fully 3D ray-tracing, non local thermal transport models) and the design of robust, realistic irradiation schemes, appropriate for polar direct drive, as can be implemented at NIF or LMJ.

In parallel, over the past two years at LULI (France), a study has begun on the feasibility of the shock ignition and has focused on fundamental issues concerning (i) the capacity to launch high-pressure shock in a large coronal plasma, and (ii) the efficiency of parametric instabilities whose the main effects are to backscatter a large amount of incident light and to produce hot electron population. The results ${ }^{16)}$ showed a moderate backscattered energy (less than $15 \%$ for total reflectivity in the focalization cone) due to the parametric instabilities (SBS and SRS), and that laser coupling for shock wave generation at intensities relevant to shock ignition $\left(10^{15} \mathrm{~W} /\right.$ $\mathrm{cm}^{2}$ ) is appropriately predicted by simulations.

Complementarily, theoretical studies have shown that a quasi-steady regime of laser plasma interaction is attained where the total laser energy absorption stays on the level of $65 \%$ in the laser intensity range $10^{15}-10^{16} \mathrm{~W} / \mathrm{cm}^{2}$. However, the relation between the collisional and collisionless processes changes significantly. This is manifested in the energy spectrum of electrons transporting the absorbed laser energy and in the spectrum of the reflected laser light. Brillouin backscattering evolves on longer time scales and dominates the reflectivities, although it is sensitive to the intensity. Filamentation and self-focusing do occur for all cases but on time scales too long to affect Raman backscattering. Simulations of the twoplasmon-decay show that in a hot, large-scale plasma, this instability develops in concomitance with stimulated Raman scattering. It is active only during the first picosecond of interaction, and then it is rapidly saturated due to plasma cavitation. The excited plasma waves extend to small wavelengths, above the standard Landau cutoff. The hot electron spectrum created by these two instabilities is relatively soft, limited to energies below $100 \mathrm{keV}$, which should not be a danger for the fuel core preheat in the shock ignition scenario. ${ }^{17)}$

\subsection{Fast ignition}

In the Fast Ignition approach to inertial fusion, a high intensity laser pulse of a few ps duration has to be conducted as deep as possible into a dense plasma where its energy is transferred into fast electrons or ions. The approach considered over the past 10 years consists of inserting a high-Z hollow cone into the spherical target shell that protects access for the ignition pulse during the implosion phase. Although feasible for demonstration purposes, this approach presents many inconveniences for future power plants because of a complicated target technology, excessively large mass of the cone and activation of its material. The original Fast Ignition approach is based on the idea of Hole Boring - creation of an empty channel in plasma by an additional intense laser pulse preceding the ignition one that has a lower intensity and longer duration than the main pulse. This idea was recently investigated in a renewed manner where the channel was bored by a long pulse, which is technologically much easier to produce, and efficient propagation of an intense short pulse within the channel was demonstrated. ${ }^{18)}$ Complementarily, a novel efficient method of hole boring in plasma with a sequence of short intense laser pulses was studied by simulations. It was shown that the use of a series of laser pulses with duration of a few 
inverse ion plasma periods enables the suppression of laser beam instabilities and to maintain the regime of relativistic transparency for a longer time. As a result, as illustrated in Fig. 3, a sequence of short laser pulses produces a much deeper and more stable channel than a single pulse with the same input energy. ${ }^{19)}$

After this phase of hole boring, ignition is produced by injecting a beam of particles in the target dense core, either electrons or ions. In electron-driven fast ignition, a main concern is the characterization of the hot electron source and effective transport of the electron beam. At Vulcan Petawatt (UK) buried layer targets are in use to estimate target heating, current densities and fast electron velocity distributions close to the fast electron source. Experiments utilize high luminosity, and spatial resolution capabilities of curved crystal spectrometers to obtain single-shot, resolve X-ray emission around the laser focal region. Current densities may be inferred from the observation of hollow atom emission lines, these unusual features originate from atomic transitions in ions with two or more inner electron shell vacancies. ${ }^{20)}$ A sensitive measure of electron beam velocity distributions is X-ray polarization within a spectral line. This information is extracted from suitable transitions using a pair of orthogonal spectrometers running at a Bragg angle of $45^{\circ}$ to separate out the polarized components. ${ }^{21}$ Finally, through imaging resonance line emission from buried layers in one and two dimensions it is possible to establish how electron heating develops within solid targets.

Using various allotropes of carbon, McKenna et al. ${ }^{22)}$ also showed recently that lattice structure affects the transport of energetic $(\mathrm{MeV})$ electrons in solids irradiated by ultraintense, short laser pulses. If fast electron-induced target heating occurs fast enough, in picoseconds or less, the material undergoes a transition through states of warm dense matter in which the background electrons are hot, but the ions remain essentially cold and in the structural arrangement they possessed in the condensed matter state, before both the ions and electrons relax to new equilibrium temperatures on tens of picosecond

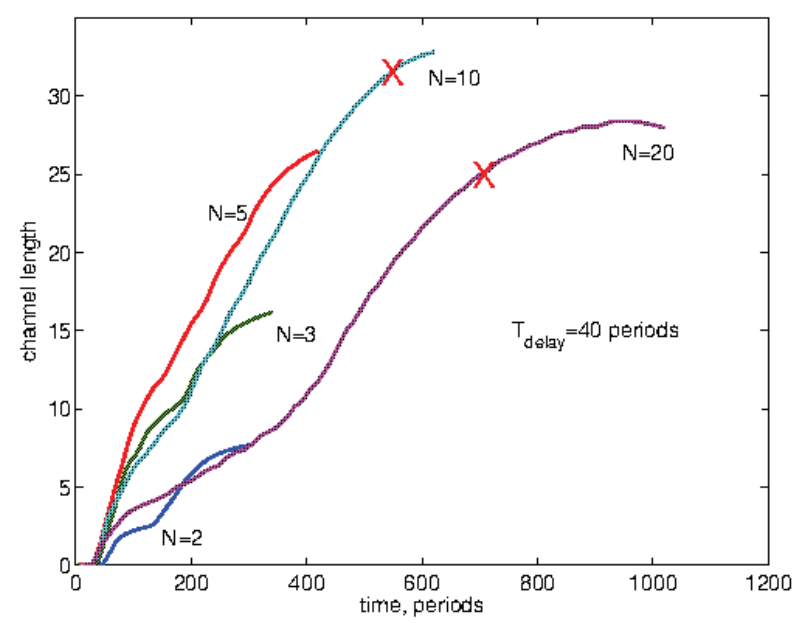

Fig. 3 Channel length (normalized to the laser wavelength) as a function of the number $N$ of laser pulses. The channel is bored in a hydrogen plasma of the density twice the critical density with a series of Gaussian laser pulses having the dimensionless amplitude $\mathrm{a}_{0}=4$, the full width at half maximum (FWHM) of 5.9 laser wavelengths and the interval between the subsequent pulses of 40 laser periods. The pulse duration is $35.3 / N$ laser periods FWHM. timescales. The study showed for the first time that the ordering of the ions in this transient state of matter plays a pivotal role in defining electrical conductivity, and therefore the onset of resistive instabilities in electron beam transport. A related study showed that fast electron transport across resistivity gradients (through proton acceleration) was inhibited due to magnetic field generation driven by the resistivity gradient. ${ }^{23)}$ Hot electron dynamics was also studied through the analysis of the characteristics of laser-accelerated protons in. ${ }^{24}$ Important differences in behavior were observed in various targets with different $\mathrm{Z}$ allowing to obtain pinched or hollow energetic electron beams. Also, Yuan et al. ${ }^{25)}$ investigated the collimating effect of self-generated resistive magnetic fields on fast electron transport in thick solid Aluminium targets (in the range $25 \mu \mathrm{m}$ to $1.4 \mathrm{~mm}$ ). Finally, transport of electrons in compressed matter could also be studied for the first time. ${ }^{26)}$

In ion-driven fast ignition, a concern is the dynamics of the focusing of laser-driven ion beams produced from concave solid targets. This was recently studied in experiments and simulations. ${ }^{27)}$ It was shown that most of the ion beam energy is observed to converge at the center of the cylindrical targets with a spot diameter of $30 \mu \mathrm{m}$. Also, it was demonstrated that unbalanced laser irradiation does not compromise the focusability of the beam.

\section{Facilities}

\subsection{LMJ and PETAL projects}

Designed to deliver $1.8 \mathrm{MJ}$ of $0.35 \mu \mathrm{m}$ light on targets with 240 beams, the laser Megajoule (LMJ), developed by The French Commissariat à l'Energie Atomique et aux Energies Alternatives (CEA), is under construction at CEA CESTA at a primary stage of $1.3 \mathrm{MJ}$ and 176 beams, and will perform HED physics experiments. One of its main goals is to obtain ignition and burn of D-T filled capsules imploded, through indirect drive scheme, inside rugby-shape hohlraums. Since the building has been commissioned in December 2008, the laser bundle assemblies of the infrared laser amplifying section are being installed and the mechanical frameworks of the target bay are being mounted around the target chamber.

Complementarily, the PETAL project will add one shortpulse (500 fs to $10 \mathrm{ps}$ ) ultra-high-power, high-energy beam (few kJ compressed energy) to the LMJ facility (see Fig. 4). PETAL will offer a combination of a very high intensity multi-petawatt beam, synchronized with the nanosecond beams of the LMJ. PETAL will expand the LMJ experimental field on HED physics and will be used for research on Fast Ignition, as a first step for the HiPER European project.

Following the decision of the implementation of PETAL on

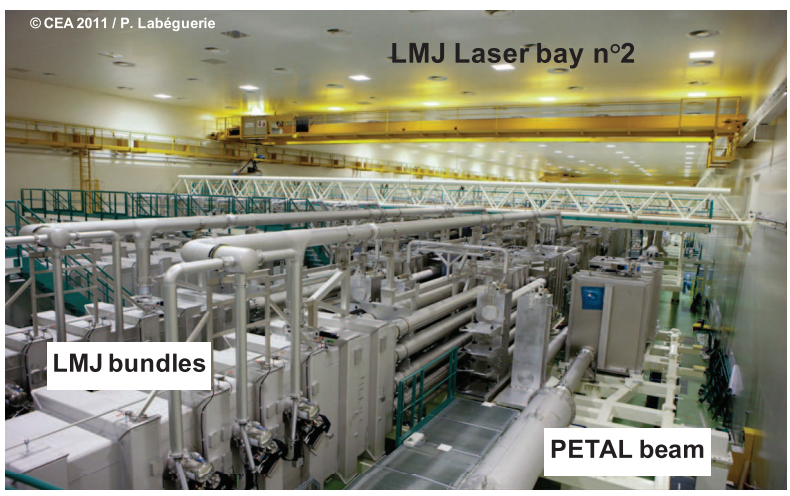

Fig. 4 View of the LMJ and PETAL laser hall. 
the LMJ, specific diagnostics adapted to PETAL capabilities have been designed to be fitted on the LMJ target chamber; this is the aim of the so-called PETAL+ program. ${ }^{28)}$ The three diagnostics have been given priority in order to characterize particles and radiation yields that can be created by PETAL. The list and general performances of these diagnostics is presented in the following table. A first set will measure the particles (protons/ions/electrons) spectrum and will also provide point projection radiography using either protons or X-rays. Another one will measure the hard X-rays lines emission in the 5 to $100 \mathrm{keV}$ range.

\begin{tabular}{llll}
\hline Type & & Range & Resolution \\
\hline Particles & Protons/ions & $0.1-200 \mathrm{MeV}$ & $10 \%$ \\
\cline { 2 - 4 } spectrometers & Electrons & $0.1-20 \mathrm{MeV}$ & $10 \%$ \\
\hline $\begin{array}{l}\text { Hard X-rays } \\
\text { spectrometer }\end{array}$ & X-rays & $5-100 \mathrm{keV}$ & $\Delta \lambda / \lambda=1 / 50 \sim 1 / 100$ \\
\hline
\end{tabular}

Aside from the PETAL+ effort, new diagnostics for X-ray imaging are also developed ${ }^{29)}$ at the Intense Laser Irradiation Laboratory group at INO-CNR (Pisa).

\section{Investigation of magnetized HED plasmas}

In the frame of ICF, in laser-produced plasma, strong magnetic fields (MG-level) are suspected not only to be ubiquitous in laser-generated, high-energy density plasmas, but also to play an important role in the laser-plasma coupling during laser irradiation and in the subsequent evolution of the heating of the target. Magnetic fields are thought to originate from crossed density and temperature gradients. Therefore, accurate modeling of electron transport in numerical codes is a great of importance. Since this last decade, new efficient algorithms of electron transport have been developed and integrated in the 2D hydro-radiative code FCI2, developed by CEA. FCI2 code takes into account resistive magneto-hydrodynamic (MHD) equations and a two-dimensional kinetic model for nonlocal transport including the effect of azimuthal magnetic fields. ${ }^{30)}$

Magnetic fields are also important for HED physics studies aiming at performing laboratory astrophysics studies since magnetic fields are a crucial ingredient in many astrophysical systems. They can range in intensity from a few micro-Gauss in the interstellar medium to giga-gauss in pulsars. Although the magnetic energy is often in equipartition with the thermal, kinetic (including turbulent), and gravitational energies, even a relatively weak, supposedly "dynamically unimportant" magnetic field can still have profound effects on the plasma. A clear example being the magneto-rotational instability which is the main responsible for the generation of turbulence and the transport of angular momentum in discs, and develops with sub-thermal fields. Moreover magnetic forces not only compete with gravity due to their long range, but can also induce strong anisotropies in the plasma. As opposed to the more quiescent unmagnetized plasmas, these are some of the reasons that magnetized plasmas are liable to a vast diversity of both small-scale phase-space instabilities, and large-scale fluid-like instabilities, which invariably lead to a redistribution of energy, and the formation of structures.

However, although magnetic fields are observed throughout galactic and intergalactic space, what is still puzzling is how they were originally created and how they became so strong. This was investigated in an experiment performed using the LULI2000 to create magnetic fields similar to those thought to be involved in the formation of the first galaxies. The team exploded a rod of carbon, similar to pencil lead, in helium gas. The explosion was designed to mimic the cauldron of plasma out of which the first galaxies formed. It was found that within a microsecond of the explosion strong electron currents and magnetic fields formed around a shock wave. When scaled to proto-galactic shocks, it is found that the measurements matched the 'magnetic seeds' predicted by theoretical studies of galaxy formation. The results closely match theories which predict that tiny magnetic fields - magnetic seeds - precede the formation of galaxies. These fields can be amplified by turbulent motions and can strongly affect the evolution of the galactic medium from its early stages. ${ }^{31}$

Further, by coupling laser-driven plasmas with an external magnetic field, as is now possible, ${ }^{32)}$ experiments can shed light on the non-linear evolution of both instabilities and dynamics of many astrophysically relevant plasma phenomena, with application to the acceleration of particles in shocks and the turbulent transport of cosmic rays, the formation of stars and protoplanetary discs in magnetized cores, magnetic reconnection, and the ejection and collimation of jets. Regarding the latter, as can be seen in Fig. 5, collimation of plasmas plumes can be obtained, dependent on applied poloidal magnetic field. ${ }^{33)}$ This has been tested successfully in another experiment performed using the ELFIE laser facility at LULI where laser produced plasma plumes generated by a $500 \mathrm{ps/}$ $50 \mathrm{~J}$ laser beam were immersed in an external magnetic field

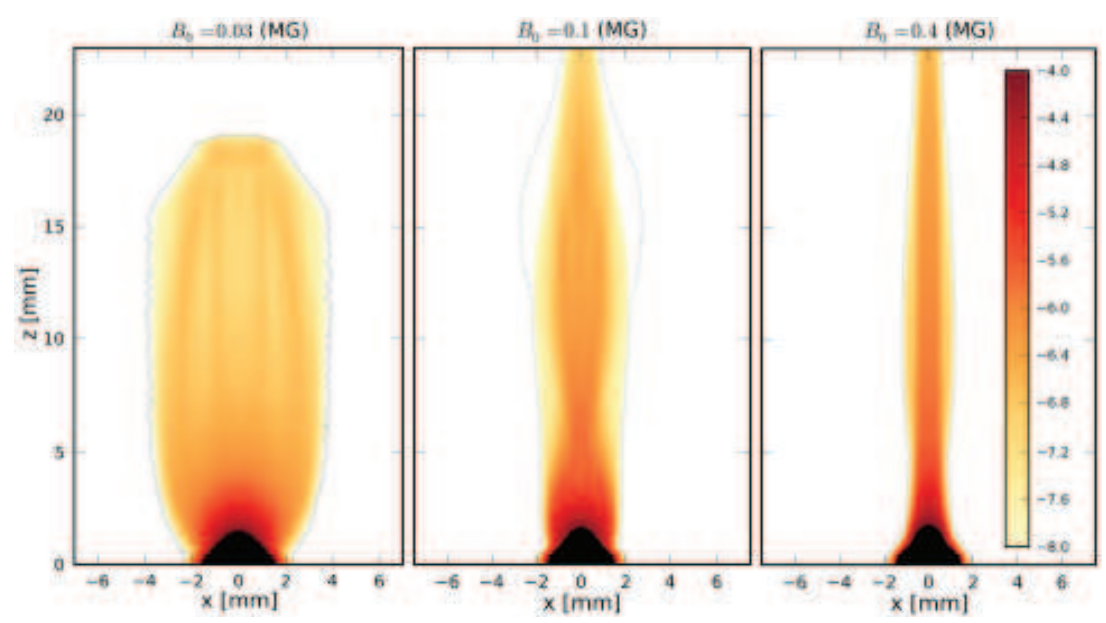

Fig. 5 Line of sight integrated density $\left(\int p d y\right)$ in $\mathrm{g} . \mathrm{cm}^{-2}$ on a logarithmic scale, $30 \mathrm{~ns}$ after the end of the laser pulse for an $\mathrm{Al}$ target and laser intensity $I=1.5 \times 10^{13} \mathrm{~W} \cdot \mathrm{cm}^{-2}$. 
of $\sim 20 \mathrm{~T}$.

\section{Summary}

In summary, we have given various examples of recent HED physics research performed in Europe, showing the breadth of the applications covered in many laboratories. This exciting research, at the forefront of both technology and science, will certainly keep expanding and allow opening up many new varied venues for scientific and societal applications.

\section{References}

1) S. Ichimaru: Rev. Mod. Phys. 54 (1982) 1017.

2) Frontiers in High Energy Density Physics: The X-Games of Contemporary Science (The National Academies Press, Washington DC, 2003).

3) E. G. Hill and S. J. Rose: High Energy Density Phys. 8 (2012) 307.

4) S. H. Glenzer and R. Redmer: Rev. Mod. Phys. 81 (2009) 1625; R. R. Fäustlin et al.: Phys. Rev. Lett. 104125002 (2010).

5) M. Harmand et al.: submitted (2012).

6) J. Alvarez Ruiz, A. Rivera, K. Mima, D. Garoz, R. GonzalezArrabal, N. Gordillo, J. Fuchs, K. Tanaka, I. Fernández, F. Briones, and J. Perlado: Plasma Phys. Contr. Fusion. in Press (2012).

7) C. Guerrero, J. Perlado, and S. Cuesta: Section: First Workshop on Fusion Technologies and the Contribution of TECHNOFUSION (2011).

8) C. Guerrero, S. Cuesta-López, and J. M. Perlado: Eur. Phys. J. Web of conferences. Proc. Seventh Conference on Inertial Fusion Sciences and Applications (IFSA 2011). in press (2012).

9) C. Guerrero, J. Perlado, and S. Cuesta: HiPER Fellow Meeting 2012. Poster Presentation (2012).

10) M. Gauthier, et al., submitted (2012).

11) R. Betti, et al.: Phys. Rev. Lett. 98 (2007) 155001.

12) S. Atzeni, A. Schiavi, and A. Marocchino: Plasma Phys. Controll.
Fusion 53 (2011) 035010.

13) X. Ribeyre, et al.: Plasma Phys. Controll. Fusion 51 (2009) 015013.

14) S. Atzeni, et al.: submitted.

15) S. Atzeni, A. Marocchino, and A. Schiavi: Phys. Plasmas 19 (2012) 090702.

16) S. D. Baton, et al.: Phys. Rev. Lett. 108 (2012) 195002.

17) O. Klimo, V. T. Tikhonchuk, X. Ribeyre, G. Schurtz, C. Riconda, S. Weber, and J. Limpouch: Phys. Plasmas 18 (2011) 082709; C. Riconda, S. Weber, V. T. Tikhonchuk, and A. Héron: Phys. Plasmas 18 (2011) 092701; S. Weber, C. Riconda, O. Klimo, A. Héron, and V. T. Tikhonchuk: Phys. Rev. E 85 (2012) 016403.

18) J. Fuchs, et al:: Phys. Rev. Lett. 105 (2010) 225001.

19) V. Mironov, et al.: Plasma Phys. Control. Fusion 54 (2012) 095008.

20) J. Colgan, et al.: in preparation.

21) N. C. Woolsey, et al.: High Energy Density Phys. 7 (2) (2011) 105-109.

22) P. McKenna, A. P. L. Robinson, D. Neely, M. P. Desjarlais, D. C Carroll, M. N. Quinn, X. H. Yuan, C. M. Brenner, M. Burza, M. Coury, et al:: Phys. Rev. Lett. 106 (2011) 185004.

23 L. A. Gizzi, S. Betti, E. Förster, D. Giulietti, S. Höfer, P. Köster, L. Labate, R. Lötzsch, A. P. L. Robinson, and I. Uschmann: Phys. Rev. ST Acc. Beams 14 (2011) 011301.

24) Y. Sentoku, E. d'Humières, L. Romagnani, P. Audebert, and J. Fuchs: Phys. Rev. Lett. 107 (2011) 135005

25) X. H. Yuan, A. P. L. Robinson, M. N. Quinn, D. C. Carroll, M. Borghesi, R. J. Clarke, R. G. Evans, J. Fuchs, P. Gallegos, L. Lancia, et al.: New J. Phys. 12 (2010) 063018.

26) F. Perez, et al.: Phys. Rev. Lett. 107 (2011).

27) S. N. Chen, et al.: Phys. Rev. Lett. 108 (2012) 055001.

28) J.-E. Ducret, S. Bastiani-Ceccotti, D. Batani, N. Blanchot, E. Brambrink, A. Casner, T. Ceccotti, A. Compant La Fontaine, E. d'Humières, et al.: submitted to Nucl. Instr. Meth. Phys. Res. A.

29) L. Labate, et al.: Rev. Sci. Inst., 2012, in press.

30) G. Schurtz, et al.: Phys. Plasma 7 (2000) 4238; Ph. Nicolai, et al.: Phys. Plasma 7 (2000) 4250.

31) G. Gregori, et al.: Nature (26 January 2012).

32) B. Albertazzi, et al:: submitted (2012).

33) A. Ciardi, et al.: submitted (2012). 